\title{
Dissecting Hirschsprung disease
}

\author{
Eberhard Passarge \\ Institut für Humangenetik, Universitätsklinikum Essen, D-45122 Essen, Germany.e-mail: eberhard.passarge@uni-essen.de
}

Published online: 15 April 2002, DOI: 10.1038/ng878

Nonsyndromic Hirschsprung disease, hitherto assumed to be a multifactorial disease with a threshold effect due to an unknown number of genes, has been genetically dissected and shown to result from an interaction between just three loci, two of which have not previously been associated with the disease. Oligogenic inheritance can explain the main aspects of its genetic epidemiology.

In complex (multifactorial or multigenic) disorders, it has not so far been possible to identify the particular contributions of individual genes, making it difficult to correlate genotype with phenotype ${ }^{1,2}$. In an accompanying paper $^{3}$, Stacey Bolk Gabriel and colleagues offer an innovative solution to this problem, and in so doing provide the first complete genetic dissection of such a multifactorial disorder. Their approach should serve as a model for the analysis of other complex diseases with nonmendelian inheritance patterns.

\section{The hallmarks of Hirschsprung \\ disease}

Hirschsprung disease (HSCR; OMIM 142623, ref. 4), also known as congenital intestinal aganglionosis, is a genetically determined, surgically correctable, neonatal intestinal obstruction syndrome (Fig. 1). It has an incidence of about 1 in 5,000 newborns. First recognized by Harald Hirschsprung in 1888 in Copenhagen, the primary cause absence of the intramural intestinal ganglion cells required for bowel peristalsis - was not recognized until 1948 (ref. 5). Their absence is due to a failure in the time-specific migration of neural-crestderived intestinal ganglion cells into the intestinal tract (refs 6,7).

Two types of Hirschsprung disease can be distinguished: short-segment (SHSCR, also called Type I HSCR), which affects the rectum and a relatively small portion of the colon and accounts for about $60-85 \%$ of cases, and the rarer long-segment (L-HSCR or Type II HSCR), which affects a longer portion of the intestine and occurs in 15-25\% of cases. About $70 \%$ of affected individuals with congenital intestinal aganglionosis have no other manifestations (nonsyndromic forms); about $30 \%$ have associated congenital malformations or an additional genetic disorder (syndromic forms $)^{8}$. Some of the syndromic forms of Hirschsprung disease involve other cell types also derived from the neural crest, such as precursors of melanocytes for cra-

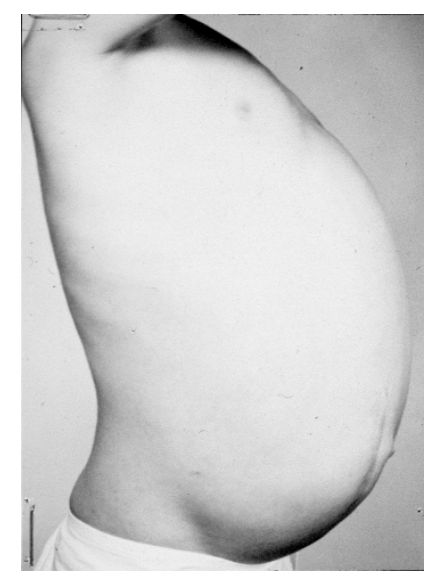

Fig. 1 The extended abdomen that is characteristic of Hirschsprung disease. It is due to the condition of megacolon that results from the absence of intestinal ganglion cells.

nial pigment formation or in cells destined to function in the sensory components of the acoustic pathway.

Early genetic studies of the familial occurrence of nonsyndromic Hirschsprung disease ${ }^{9-11}$ invoked a multigenic model to explain the usually nonmendelian inheritance pattern, with an average risk of recurrence in siblings of $3-4 \%$ (but reaching $17 \%$ in certain families), about 200-fold higher than the risk in the general population. The sex ratio is four males to one female. The proportion of affected sibs is higher when the index patient is female. This is thought to be the result of a greater contribution of genetic factors required for the manifestation of the disease in females, known as the Carter effect ${ }^{7}$. Familial incidence is lower for S-HSCR than for L-HSCR, and the genetic relationship between the two forms of the disorder is still not understood.

\section{Genetic analysis}

Normal development and migration of the neural crest-derived intestinal ganglion cells involves genes in three different signaling pathways ${ }^{12-15}$ : (i) the RET receptor tyrosine kinase pathway with genes encoding the RET receptor and its ligand, the glial cell line-derived neurotrophic factor (GDNF); the endothelin type $B$ receptor pathway with the EDNR receptor and its ligand, endothelin-3 (EDN3); and (iii) SOX10-mediated transcription ${ }^{6-8}$. All told, mutations in eight partially-interdependent genes are associated with Hirschsprung disease (see table).

None of the mutations is fully penetrant (see table) and they have varying effects on the length of the aganglionic segment of intestine. Dominant mutations in RET have been found in about $50 \%$ of familial patients with Hirschsprung disease and about $15-35 \%$ of isolated cases, with a penetrance of $50 \%$ in females and $70 \%$ in males. Mutations in EDNRB are recessive, with reduced penetrance (30-80\%). Mutations in any gene are detected in only about $60 \%$ of affected individuals and in an even lower percentage of the patients with S-HSCR studied by Bolk Gabriel et al. ${ }^{3}$.

\begin{tabular}{llll}
\hline & \multicolumn{2}{c}{ Genes implicated in the etiology of Hirschsprung disease } \\
\hline Gene & Location & Main effect & Penetrance \\
RET & $10 q 11.2$ & dominant, loss-of-function & $50-72 \%$ \\
GDNF & $5 p 13.1$ & dominant/recessive & unknown \\
EDNRB & $13 q 22$ & recessive & $30-85 \%$ \\
EDN3 & $20 q 13$ & recessive & unknown \\
SOX10 & $22 q 13$ & dominant/recessive & $>80 \%$ \\
ECE1 & $1 \mathrm{p} 36$ & dominant/recessive & unknown \\
NTN & $19 \mathrm{p} 13$ & unknown & unknown \\
SIP1 & $2 q 22$ & sporadic & unknown \\
\hline
\end{tabular}

Most effects seem to be interdependent, and mutant alleles usually do not segregate in a mendelian pattern. 


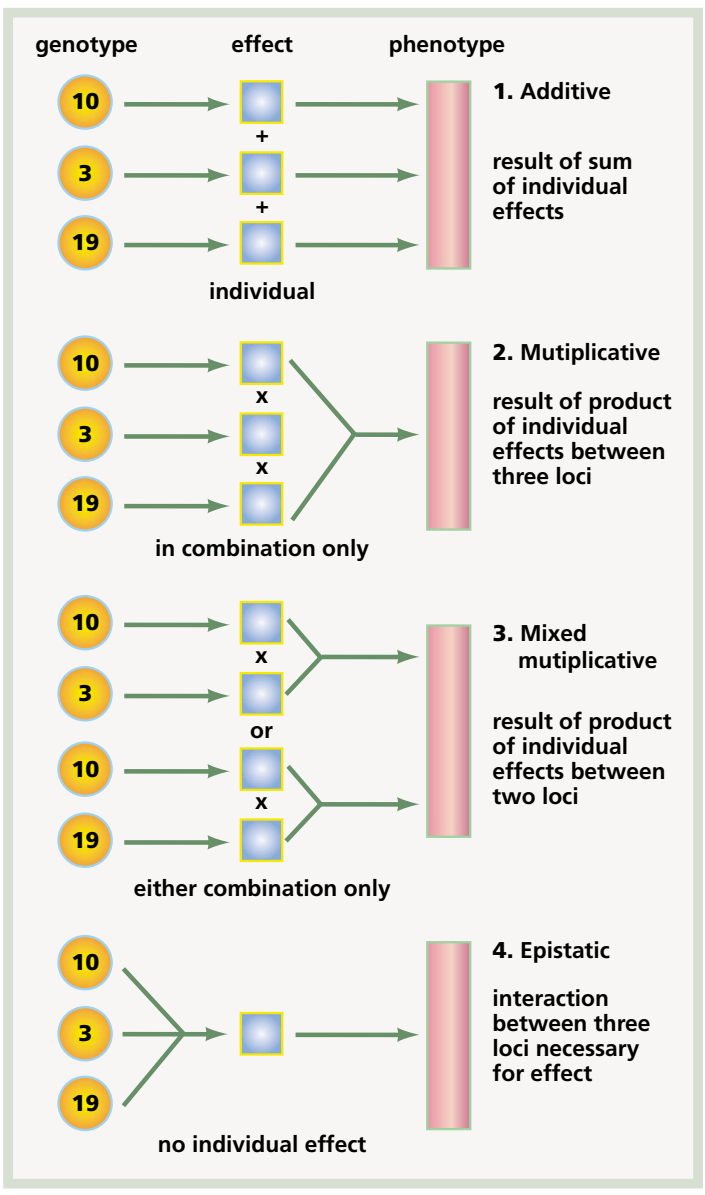

BOB CRIMI

\section{And then there were three}

Bolk Gabriel et al. ${ }^{3}$ studied 49 families with S-HSCR, in which there were a total of 67 distinct affected sib pairs and a total of 106 affected individuals all with nonsyndromic Hirschsprung disease. Using microsatellite markers, they undertook a genome-wide linkage scan covering more than $92 \%$ of the human genome. Multipoint nonparametric linkage analysis detected significant allele-sharing through identity-by-descent among affected sibs in three chromosomal regions: on 10q11 at the RET locus, which encodes the RET receptor, and in two regions previously unknown to be related to Hirschsprung disease $-3 \mathrm{p} 21$ and 19q12. The main gene conferring susceptibility was confirmed to be RET through the
Fig. 2 Four models for the interaction of three loci at 10q11, $3 p 21$ and $19 q 12$ in the genetic dissection of short-segment Hirschsprung disease.

identification of codingregion mutations in 17 of 43 linked families. The failure to identify codingregion mutations in some of the RET-linked families suggests the existence of mutations in regulatory regions. In contrast to multigenerational HSCR families, where mutations occurred throughout the gene, mutations in RET were distributed nonrandomly in the region encoding extracellular domains of the receptor. The reason for this difference and how it might relate to the differences between S-HSCR and LHSCR remains poorly understood. Surprisingly, Bolk Gabriel et al. ${ }^{3}$ found a distorted parental transmission of susceptibilty alleles of RET: 21 maternal and 6 paternal transmissions. This parent-of-origin effect might explain the unusual sex ratio of 4:1 (males to females) in Hirschsprung disease.

\section{Multiplicative effects}

Bolk Gabriel et al. ${ }^{3}$ assessed the magnitude of the genetic effects by comparing how the three loci might interact. They used four models: additive, multiplicative, mixed multiplicative and epistatic (Fig. 2). The additive model assumes an individual effect of any single locus that can lead to HSCR. The effects of the three loci (at 10q11,3p12 and 19q12) are simply added. This yielded a lod score of 7.15. The multiplicative model assumes that all three loci are involved. Their individual contributions are multiplied, which yielded a lod score of 13.07. A mixed multiplicative model in which interactions occur between 10q11 and 3p21 in some families and $10 \mathrm{q} 11$ and $19 \mathrm{q} 12$ in others yielded a lod score of 12.22, and remains a possibility. In the model assuming epistatic effects between the three loci, a lod score of 13.09 was obtained. However, this model required an additional fourth parameter and was considered less likely than the multiplicative model. Thus, all three loci seem to be involved. Moreover, the three are necessary and sufficient and other loci are unlikely to be involved. A multiplicative effect of the three loci is consistent with the recurrence risk in sibs, the disease incidence (population risk), and transmission distortion, and thus appears to be the most likely model.

The new study is a major advance in understanding nonsyndromic Hirschsprung disease, by demonstrating oligogenic inheritance attributable to just three loci. The approach used by Bolk Gabriel et al. ${ }^{3}$ should serve as a model for dissecting other complex genetic diseases. However, a prerequisite for such an analysis is the careful clinical characterization of the disease to be studied.

1. Lander E.S. \& Kruglyak, L. Nature Genet. 11, 241-247 (1995).

2. Altmüller, J. et al. Am. J. Hum. Genet. 69, 936-950 (2001).

3. Bolk Gabriel, S. et al. Nature Genet. 31, (2002); advance online publication, (DOI: 10.1038/ng868).

4. McKusick, V.A. Mendelian Inheritance in Man. A Catalog of Human Genes and Genetic Disorders. 12th edn (Johns Hopkins University Press, Baltimore, 1998).

5. Zuelzer, W.W. \& Wilson J.L. Am. J. Dis. Childr. 75, 40-64 (1948).

6. Chakravarti, A. \& Lyonnet, S. in The Metabolic \& Molecular Bases of Inherited Disease. 8th edn (eds Scriver C.R., Beaudet, A.L., Sly, W. \& Valle, D.) 6231-6255 (McGraw-Hill, New York, 2001).

7. Passarge, E. in Emery \& Rimoin's Principles and Practice of Medical Genetics. 4th edn (eds Rimoin, D.L., Connor, J.M., Pyeritz, R.E. \& Korf, B.R.) 1747-1759 (Churchill-Livingstone, Edinburgh, 2002).

8. Amiel, J. \& Lyonnet, S. J. Med. Genet. 38, 729-739 (2001).

9. Bodian, M. \& Carter C.O. Ann. Hum. Genet. 29, 261-277 (1963).

10. Madsen, C.M. Hirschsprung's Disease (Munksgaard, Copenhagen, 1964).

11. Passarge, E. N. Engl. J. Med. 276, 138-143 (1967).

12. Lyonnet, S. et al. Nature Genet. 4, 346-350 (1993).

13. Angrist, M. et al. Nature Genet. 4, 351-356 (1993).

14. Wakamatsu, N. et al. Nature Genet. 27, 369-370 (2001).

15. Hofstra, R.M.W. et al. Hum. Mutat. 15, 418-429 (2000). 